\title{
Stark Resonances in Disordered Systems
}

\author{
Vincenzo Grecchi ${ }^{1}$, Marco Maioli ${ }^{2,3}$ and Andrea Sacchetti ${ }^{3}$ \\ ${ }^{1}$ Dipartimento di Matematica, Università degli Studi di Bologna, I-40127 Bologna, Italy \\ ${ }^{2}$ Istituto di Matematica, Università degli Studi della Basilicata, I-85100 Potenza, Italy \\ ${ }^{3}$ Dipartimento di Matematica Pura ed Applicata, Università degli Studi di Modena, \\ I-41100 Modena, Italy
}

Received July 23, 1991; in revised form November 7, 1991

\begin{abstract}
By slightly restricting the conditions given by Herbst and Howland, we prove the existence of resonances in the Stark effect of disordered systems (and atomic crystals) for large atomic mean distance. In the crystal case the ladders of resonances have the Wannier behavior for small complex field.
\end{abstract}

\section{Introduction}

In 1981 Herbst and Howland had provided a setup for the definition of resonances in the case of one-dimensional regular crystals as well as of disordered systems in a uniform electric field. They omitted the existence proof.

Starting from such a construction, and slightly restricting the general conditions of Herbst and Howland (1981), we provide existence theorems for resonances in disordered systems in the regime of large distance between atoms at small fixed electric field. This result adds to and completes the recent results on the crystal case given by Bentosela and Grecchi (1991), Buslaev and Dmitrieva (1990) and Combes and Hislop (1991) (see also Agler and Froese (1985) for the case of large electric field). Our proof (see Sect. 2) is very general and refers to standard techniques of eigenvalue stability. If our treatment is specialized to the case of an ordered atomic crystal (see Sect. 3), we obtain exact ladders of resonances, that are uniquely associated with the complex field states of Avron (1979) and Bentosela et al. (1988) and with the single-band approximation of Wannier.

As a particular case, we consider a model that in the zero field case coincides with the classical Lamé problem, which, for integral values of a parameter, gives rise to a finite number of bands. In the last case, but for small positive field, we prove the existence of a ladder for each Lamé finite band, as suggested by Avron (1982). For a study of the quasi regular case by perturbation theory methods we refer to the paper of Nenciu and Nenciu (1989). 
Finally, let us note that in the disordered system case the absolutely continuous spectrum disappears, almost surely, at zero field (Kirsch et al. (1985)), so that no resonances exist in such a limit (but, perhaps, bound states).

\section{Stark States for Disordered Systems}

Let us consider the Hamiltonian of the form

$$
H:=p^{2}+V_{0},
$$

where $V_{0}$ is the atomic type potential defined by a real-valued function $u(x)$ on $\mathbf{R}$ such that:

a) $u(x)$ admits analytical translation, $x \rightarrow x-i \theta$, and dilation, $x \rightarrow x e^{i \varphi}$, for $|\theta|<\theta_{0}$ and $|\varphi|<\varphi_{0}$ (Herbst (1979)).

b) for any positive $\theta<\theta_{0}, \exists C, r>0, q>1$ such that

$$
|u(z)| \leqq C|z|^{-q}, \quad \forall z \quad \text { such that }|\mathfrak{J} z|<\theta \quad \text { and } \quad|\mathfrak{R} z|>r .
$$

Then (1) defines a self-adjoint operator (still denoted $H$ ) on the domain $D\left(p^{2}\right)$, where $\Sigma_{\text {ess }}(H)=[0,+\infty)$ and $\Sigma_{d}(H) \subset(-\infty, 0)$ has multiplicity 1 .

Finally, we assume that:

c) $\Sigma_{d}(H)$ is not empty, i.e. there exist at least $n, n \geqq 1$, negative eigenvalues of the operator $H: \lambda_{1}<\lambda_{2}<\cdots<\lambda_{n}<0$.

The class of such potentials defined by a), b) and c) is clearly not void (see for instance formula (37)).

Now, adding in (1) the term $F x$ and performing the analytical translation $x \rightarrow x-i \theta, 0<\theta<\theta_{0}$, we obtain the operator formally defined by

$$
H_{0, F}^{\theta}:=p^{2}+V_{0}^{\theta}+F x-i F \theta
$$

and belonging to the class of Stark models, $V_{0}^{\theta}$ is the potential defined by $u(x-i \theta)$. For $F>0$ real we have that $\Sigma_{\text {ess }}\left(H_{0, F}^{\theta}\right) \subseteq-i F \theta+\mathbf{R}$ and that, for $F$ small enough, $\Sigma_{d}\left(H_{0, F}^{\theta}\right)$ consists of $n \theta$-independent non-degenerate eigenvalues $\lambda_{j}^{0, F}$ close to $\lambda_{j}, j=1,2, \ldots, n$, having imaginary part of order $O\left(F^{\infty}\right)$. This follows from Theorem III.3 in Herbst (1979), concerning a Hamiltonian of the type (3) submitted to an analytical dilation, and from the coincidence of resonances defined by the analytical dilation with respect the analytical translation method (see, e.g., Sigal (1988)). These arguments work for $F$ complex too and, assuming $\mathfrak{I} F>0$ for sake of simplicity, we have that $H_{0, F}^{\theta}$ has compact resolvent (it follows from Theorem II.3 in Herbst (1979) and from the relative compactness of the term $V_{0}^{\theta}$ ), its eigenvalues are $\theta$-independent and the stability as $F \rightarrow 0$ of the $n$ eigenvalues of $H$ holds again uniformly for $F$ in any sector $0 \leqq \arg F \leqq \bar{\delta}$. Moreover we have $\mathfrak{I} \lambda_{j}^{0, F}=c_{0} \cdot \mathfrak{I} F+$ $O\left(F^{2}\right), c_{0}$ is the first perturbative coefficient.

We summarize these results in the following:

Theorem 1. Let $\theta, 0<\theta<\theta_{0}$ be fixed. We have that:

i) for any $d_{1}>0$ and any $j, j=1,2, \ldots, n$, there exists $F_{0}>0$ such that $H_{0, F}^{\theta}$ has only one $\theta$-independent isolated eigenvalues $\lambda_{j}^{0, F}$ in the set $\left\{\lambda \in \mathbf{C}|| \lambda-\lambda_{j} \mid<d_{1}\right\}$ for any $F \in\left\{z \in \mathbf{C}|| z \mid \leqq 2 F_{0}, 0 \leqq \arg z \leqq \delta\right\}$. Moreover, for $\delta>0$ small enough we have that $\left|\mathfrak{J} \lambda_{j}^{0, F}\right|<\frac{1}{4}|F| \theta$. 
ii) if $F$ is real, $\Sigma_{\mathrm{ess}}\left(H_{0, F}^{\theta}\right) \subseteq-i F \theta+\mathbf{R}$ and the eigenvalues $\lambda_{j}^{0, F}$ have imaginary part of order $O\left(F^{\infty}\right)$;

iii) if $\mathfrak{I} F>0, H_{0, F}^{\theta}$ has compact resolvent, hence $\Sigma_{\text {ess }}\left(H_{0, F}^{\theta}\right)=\varnothing$.

We regard the operator $H_{0, F}^{\theta}$ as a limit problem for the class of operators $\left\{H_{\beta, F}^{\theta}\right\}_{\beta \geqq 0}$, as $\beta \downarrow 0$, of the Stark-Wannier type

$$
H_{\beta, F}^{\theta}:=p^{2}+V_{\beta}^{\theta}+F x-i F \theta
$$

for $F$ in the sets $\Omega_{\delta, f}$ and $\Theta_{\varepsilon, f}$, where

$$
\Omega_{\delta, f}:=\{F \in \mathbf{C}|f \leqq| F \mid \leqq 2 f, 0 \leqq \arg F \leqq \delta\}
$$

and

$$
\Theta_{\varepsilon, f}:=\{F \in \mathbf{C}|f \leqq| F \mid \leqq 2 f, \varepsilon \leqq \arg F \leqq \pi / 2\},
$$

where $0<f<F_{0}, 0<\varepsilon<\delta, \delta$ and $F_{0}$ as in Theorem 1 . Here $V_{\beta}^{\theta}$, for $\beta>0$, is the type multiple-atomic potential defined by $u_{\beta}(x-i \theta)$, where

$$
u_{\beta}(z):=\sum_{m \in \mathbf{Z}} u\left(z-\beta^{-1} X_{m}\right), \quad \beta>0,|\mathfrak{I} z|<\theta_{0},
$$

each $X_{m}, m \in \mathbf{Z}$, can represent the position of the $m^{\text {th }}$ atom. As a model of disordered system we consider $X_{m}(\omega), \omega$ in a probability space $(\Omega, \mathscr{F}, P)$, the points of a Poisson ensemble on $\mathbf{R}$, with mean density 1 , where we assume an ordering

$$
\cdots<X_{-1}<X_{0}<X_{1}<\cdots \text {. }
$$

In the following, for sake of definiteness, we assume $X_{0}(\omega)=0$ for any $\omega$ (otherwise we translate $x \rightarrow x+\beta^{-1} X_{0}$ ). We have that, $P$-almost surely, $u_{\beta}(z)$ is finite and real-analytic in the strip $|\mathfrak{I} z|<\theta_{0}$. Moreover, the following bound holds for $\beta$ small enough

$$
\left|u_{\beta}(x-i \theta)\right| \leqq C_{\beta} \log (\beta|x-i \theta|+2)+C_{0}, \quad|\theta|<\theta_{0}, P \text {-a.s. }
$$

where $C_{0}, C_{\beta}>0$ and $C_{\beta} \rightarrow 0$ as $\beta \downarrow 0$. Indeed, let $v(z):=\left(|z|^{2}+1\right)^{-q / 2}, q>1$ as in (2), satisfying the hypothesis of Corollary 3.5 in Herbst and Howland (1981), hence

$$
\bar{v}(z):=\sum_{m \in \mathbf{Z}} v\left(z-X_{m}(\omega)\right)
$$

is finite, $P$-a.s., and such that for some $\bar{C}$

$$
|\bar{v}(z)| \leqq \bar{C} \log (|z|+2)
$$

Therefore

$$
\begin{aligned}
\left|u_{\beta}(x-i \theta)\right| & =\left|\sum_{m} u\left(x-i \theta-\beta^{-1} X_{m}(\omega)\right)\right| \text { as } B \rightarrow 0 \\
& \leqq C \sum_{m \neq 0} v\left(x-i \theta-\beta^{-1} X_{m}(\omega)\right)+C_{0} \\
& \leqq C \beta^{q} \sum_{m \neq 0}\left[\left|(x-i \theta) \beta-X_{m}(\omega)\right|^{2}+\beta^{2}\right]^{-q / 2}+C_{0} \\
& \leqq \bar{C} C \beta^{q} \log (|x-i \theta| \beta+2)+C_{0} .
\end{aligned}
$$


In particular, from geometric estimates, a type (7) estimate holds for $\frac{d u_{\beta}(z)}{d z}$ too. Moreover, from similar arguments, we have that for each $x \in \mathbf{R}, m \in \mathbf{Z}$ and $\theta,|\theta|<\theta_{0}$,

$$
\lim _{\beta \downarrow 0} u_{\beta}\left(x-i \theta+\beta^{-1} X_{m}\right)=u(x-i \theta), \quad P \text {-a.s. }
$$

Now, let us stress that, for $F$ real, $H_{\beta, F}^{\theta}$ satisfies, $P$-a.s., the hypotheses of Theorem 2.1 in Herbst and Howland (1981); hence, it has discrete spectrum (if it is not empty) lying in the strip $\{z \mid z \in \mathbf{C}, 0 \geqq \mathfrak{I} z>-F \theta\}$ and its essential spectrum is contained in the line $-i F \theta+\mathbf{R}$. Moreover, for $F$ complex and $\mathfrak{I} F \neq 0, H_{\beta, F}^{\theta}$ has compact resolvent (see Avron (1979)), hence $\Sigma_{\text {ess }}\left(H_{\beta, F}^{\theta}\right)=\varnothing$.

We are going to prove the following theorem:

Theorem 2. Let $\theta$, with $0<\theta<\theta_{0}$, be fixed and $F_{0}$ and $\delta$ as in Theorem $1 i$. Then for any $f, 0<f<F_{0}$, there exists $\bar{\beta}>0$ such that, $\forall F \in \Omega_{\delta_{\text {,f }}}$ and $\forall \beta \in(0, \bar{\beta}]$ the operator $H_{\beta, F}^{\theta}$ admits, $P$-a.s., an eigenvalue $\lambda_{j}^{\beta, F}+F \beta^{-1} X_{m}(\omega)$ at least, for some $m \in Z$, where $\lambda_{j}^{\beta, F} \equiv \lambda_{j}^{\beta, F}(\omega)$ tends to $\lambda_{j}^{0, F}$ as $\beta \downarrow 0, j=1,2, \ldots, n$, uniformly with respect to $F$ in $\Omega_{\delta, f}$.

Proof of Theorem 2. P-a.s. choose $m=m(\omega)$ s.t. (11) holds uniformly w.r.t. $\omega$. Since

$$
\Sigma_{d}\left(H_{\beta, F}^{\theta}\right)=\Sigma_{d}\left(T_{\beta^{-1} X_{m}(\omega)} H_{\beta, F}^{\theta} T_{\beta^{-1} X_{m}(\omega)}^{-1}\right)+F \beta^{-1} X_{m}(\omega)
$$

where $T_{\alpha}$ is the translation operator $x \rightarrow x+\alpha$, we can assume, without loss of generality, $m=0$. Moreover, in the following, will be implicitly assumed the dependence of $\lambda_{j}^{\beta, F}$ on $\omega$.

Now, following Herbst and Howland (1981) let us consider the following representation for the resolvent

$$
\left[\hat{H}_{\beta, F}^{\theta}-\lambda\right]^{-1}=S_{\beta}(\lambda)\left[1+Q_{\beta}(\lambda) S_{\beta}(\lambda)\right]^{-1}, \quad \beta \geqq 0, \mathfrak{I} \lambda>0,
$$

where $S_{\beta}(\lambda) \equiv S_{\beta, F}(\lambda, \theta ; \gamma)$ is the inverse operator defined by $\hat{H}_{\beta, F}^{\theta}+Q_{\beta}(\lambda)-\lambda$, $\hat{H}_{\beta, F}^{\theta}:=H_{\beta, F}^{\theta}+i F \theta$, and $Q_{\beta}(\lambda) \equiv Q_{\beta, F}(\lambda, \theta ; \gamma)$ is the bounded operator defined by the function of $x \in \mathbf{R}$ given by

$$
\begin{aligned}
Q_{\beta}(\lambda)(x):= & \chi_{(1 / f,+\infty)}\left\{\frac{\left(\lambda-V_{\beta}^{\theta}\right)^{2}}{4 F x}-\frac{F^{1 / 2}}{2 x^{1 / 2}}+\frac{\lambda-V_{\beta}^{\theta}}{4 x^{3 / 2} F^{1 / 2}}-\frac{1}{2 F^{1 / 2} x^{1 / 2}} \frac{d V_{\beta}^{\theta}}{d x}\right\} \\
& +\chi_{(-\infty,-1 / f)}\left\{\frac{\left(\lambda-V_{\beta}^{\theta}\right)^{2}}{4 F x}-\frac{F^{1 / 2}}{2 i|x|^{1 / 2}}+\frac{i\left(\lambda-V_{\beta}^{\theta}\right)}{4|x|^{3 / 2} F^{1 / 2}}-\frac{i}{2 F^{1 / 2}|x|^{1 / 2}} \frac{d V_{\beta}^{\theta}}{d x}\right\} \\
& -\chi_{[-1 / f,+1 / f]}\left(F x+V_{\beta}^{\theta}-\lambda\right)+\gamma \chi_{[-1 / 2 f,+1 / 2 f]}, \quad \mathfrak{I} \lambda>0 .
\end{aligned}
$$

Here $\chi_{[a, b]}$ is the characteristic function on the interval $a \leqq x \leqq b$ and $\gamma \in \mathbf{R}^{+}$is a suitable constant whose meaning will be discussed in the following lemma.

Lemma 3. For any compact subset $\Gamma$ of $\{\lambda \in \mathbf{C}: \mathfrak{I} \lambda>0$ and $\mathfrak{R} \lambda \leqq 0\}$ there exist $\bar{\beta}>0$ and $\gamma$ such that $S_{\beta, F}(\lambda, \theta ; \gamma)$ is uniformly bounded for $(\lambda, \beta, F) \in \Lambda_{\bar{\beta}, f}:=\Gamma \times[0, \bar{\beta}] \times \Omega_{\delta, f}$.

Proof of Lemma 3. Let $\phi_{\gamma}^{+}$and $\phi_{\gamma}^{-}$be the solutions of

$$
\left[p^{2}+F x+V_{\beta}^{\theta}+Q_{\beta}(\lambda)-\lambda\right] \phi_{\gamma}^{ \pm}=0
$$

coincident, respectively, with $\phi^{+}, \phi^{+} \in L^{2}\left(\left[f^{-1},+\infty\right)\right)$, and $\phi^{-}, \phi^{-} \in L^{2}\left(\left(-\infty,-f^{-1}\right]\right)$, 
where $\phi^{ \pm}$are the WKB solutions of $\left[p^{2}+F x+V_{\beta}^{\theta}-\lambda\right] \phi=0$ for large $|x|$, i.e.:

$$
\phi^{+}(x)=\exp \left\{-\left[\frac{2}{3} F^{1 / 2} x^{3 / 2}-\frac{\lambda x^{1 / 2}}{F^{1 / 2}}+\frac{1}{2} \int_{0}^{x} V_{\beta}^{\theta}(t)(t F)^{-1 / 2} d t\right]\right\}
$$

and

$$
\phi^{-}(x)=\exp \left\{-\left[\frac{2}{3 i} F^{1 / 2}(-x)^{3 / 2}-\frac{i \lambda(-x)^{1 / 2}}{F^{1 / 2}}-\frac{i}{2} \int_{0}^{x} V_{\beta}^{\theta}(t)(-t F)^{-1 / 2} d t\right]\right\} .
$$

Let $W \equiv W_{\beta, F}(\lambda, \theta ; \gamma)$ be the Wronskian of $\phi_{\gamma}^{ \pm}$. First of all, we prove that there exist $\bar{\beta}, \gamma$ and $\mu>0$ such that $|W|>\mu$ for any $(\lambda, \beta, F) \in \Lambda_{\bar{\beta}, f}$. Let

$$
\left(\begin{array}{l}
\phi^{-} \\
\psi^{-}
\end{array}\right),\left(\begin{array}{l}
1 \\
x
\end{array}\right),\left(\begin{array}{c}
e^{\sqrt{\gamma} x} \\
e^{-\sqrt{\gamma} x}
\end{array}\right),\left(\begin{array}{l}
1 \\
x
\end{array}\right),\left(\begin{array}{l}
\phi^{+} \\
\psi^{+}
\end{array}\right)
$$

be, respectively, the linearly independent solutions of (15) in the intervals $\left(-\infty,-f^{-1}\right],\left(-f^{-1},-(2 f)^{-1}\right),\left[-(2 f)^{-1},(2 f)^{-1}\right],\left((2 f)^{-1}, f^{-1}\right),\left[f^{-1},+\infty\right)$, where $\psi^{ \pm}=\phi^{ \pm} \int_{ \pm f^{-1}}^{x}\left[\phi^{ \pm}\right]^{-2} d x$. Hence, we have

$$
\phi_{\gamma}^{ \pm}=c_{ \pm} e^{\sqrt{\gamma} x}+d_{ \pm} e^{-\sqrt{\gamma} x}
$$

in the interval $\left[-(2 f)^{-1},(2 f)^{-1}\right]$, where $c_{ \pm}$and $d_{ \pm}$are obtained by matching conditions on the solutions $\phi_{\gamma}^{ \pm}$. In particular, one obtains

$$
\begin{aligned}
& c_{ \pm}=\frac{-1}{2 \sqrt{\gamma}} e^{\mp \sqrt{\gamma} /(2 f)} \phi^{ \pm}\left( \pm f^{-1}\right)\left\{\left(1 \mp \frac{\sqrt{\gamma}}{2 f}\right) \Psi^{\prime}\left( \pm f^{-1}\right)-\sqrt{\gamma}\left[1 \mp \Psi^{\prime}\left( \pm f^{-1}\right)\right]\right\}, \\
& d_{ \pm}=\frac{-1}{2 \sqrt{\gamma}} e^{ \pm \sqrt{\gamma} /(2 f)} \phi^{ \pm}\left( \pm f^{-1}\right)\left\{\left(1 \pm \frac{\sqrt{\gamma}}{2 f}\right) \Psi^{\prime}\left( \pm f^{-1}\right)+\sqrt{\gamma}\left[1 \mp \Psi^{\prime}\left( \pm f^{-1}\right)\right]\right\},
\end{aligned}
$$

where $\phi^{ \pm \prime}\left( \pm f^{-1}\right)=-\Psi^{\prime}\left( \pm f^{-1}\right) \phi^{ \pm}\left( \pm f^{-1}\right)$ and

$$
\begin{aligned}
\Psi^{\prime}\left(f^{-1}\right) & =\sqrt{\frac{f}{F}}\left[\frac{F}{f}+\frac{1}{2} V_{\beta}^{\theta}\left(f^{-1}\right)-\frac{\lambda}{2}\right], \\
\Psi^{\prime}\left(-f^{-1}\right) & =-i \sqrt{\frac{f}{F}}\left[\frac{F}{f}+\frac{1}{2} V_{\beta}^{\theta}\left(-f^{-1}\right)-\frac{\lambda}{2}\right],
\end{aligned}
$$

where ' denotes $\frac{d}{d x}$. Since $\mathfrak{R} \lambda \leqq 0$ and $f$ is fixed we can choose $\bar{\beta}>0$ such that in the term $V_{\beta}^{\theta}\left( \pm f^{-1}\right)$, for any $\beta<\bar{\beta}$, the contribution given by the overlapping between the first well and the other ones can be neglected, hence

$$
\left|\Psi^{\prime}\left( \pm f^{-1}\right)\right| \geqq 1-\frac{\left|u\left( \pm f^{-1}-i \theta\right)\right|}{2} \geqq \frac{1}{2}
$$

if $f$ is small enough. Then, we have that the leading term of $|W|=\left|2 \sqrt{\gamma} d_{+} d_{-}\left[\frac{c_{-}}{d_{-}}-\frac{c_{+}}{d_{+}}\right]\right|$ 
satisfies the following estimate for some $\mu>0$ :

$$
\begin{aligned}
& \left|\frac{\sqrt{\gamma}}{8 F^{2}} \phi^{+}\left(f^{-1}\right) \phi^{-}\left(-f^{-1}\right) \Psi^{\prime}\left(f^{-1}\right) \Psi^{\prime}\left(-f^{-1}\right) e^{\sqrt{\gamma} / f}\right| \\
& \quad \geqq \frac{\sqrt{\gamma}}{32 f^{2}} \exp \left[\frac{\sqrt{\gamma}}{f}-\frac{2}{3 f}+\frac{\Re \lambda}{f}-\frac{\mathfrak{I} \lambda}{f}-\frac{1}{\sqrt{f}} \mathfrak{R} \int_{0}^{\infty} \frac{u(x-\theta)}{\sqrt{x}} d x\right]>\mu
\end{aligned}
$$

choosing $\gamma$ large enough and positive.

Here (15) has no solutions in $L^{2}(\mathbf{R})$ for $(\lambda, \beta, F) \in \Lambda_{\bar{\beta}, f}$, moreover $W^{-1}$ is uniformly bounded in $\Lambda_{\bar{\beta}, f}$.

Now, we have that $S_{\beta}(\lambda)=W^{-1} A$, where $A \equiv A_{\beta, F}(\lambda, \theta ; \gamma)$ is the operator

$$
[A f](x):=\int_{-\infty}^{+\infty} G_{\beta, F}(\lambda, \theta, \gamma ; x, y) f(y) d y,
$$

where $G_{\beta, F}(\lambda, \theta, \gamma ; x, y):=\phi_{\gamma}^{+}\left(x_{>}\right) \cdot \phi_{\gamma}^{-}\left(x_{<}\right), x_{>}=\max (x, y)$ and $x_{<}=\min (x, y)$.

Adapting to our case the proof of Proposition A.1 given in Herbst and Howland (1981), one can prove that $A$ is a uniformly bounded operator for $(\lambda, \beta, F) \in \Lambda_{\bar{\beta}, f}$ (let us stress that $f$ is small enough but fixed and that $V_{\beta}^{\theta}$ satisfies the bound (7) where, as $\beta \downarrow 0$, this estimate improves). Hence, Lemma 3 follows.

Now, let $\Gamma$ be chosen such that $\lambda_{j}^{0, F}+F \theta \in \Gamma$ for any $F \in \Omega_{\delta, f}$ and any $j, j=$ $1,2, \ldots, n$, i.e.:

$$
\Gamma:=\left\{z \in \mathbf{C}: \frac{f \theta}{2} \leqq \mathfrak{I} z \leqq \frac{5 f \theta}{2} \text { and } \lambda_{1}-1 \leqq \mathfrak{R} z \leqq 0\right\} .
$$

Lemma 4. Let $\Gamma$ be as in (23) and $\gamma$ as in Lemma 3, then $Q_{\beta}(\lambda) S_{\beta}(\lambda)$ converges in norm to $Q_{0}(\lambda) S_{0}(\lambda)$ as $\beta \downarrow 0$ uniformly with respect to $(\lambda, F) \in \Gamma \times \Omega_{\delta, f}$.

Proof of Lemma 4. Let $N \in \mathbf{R}^{+},(\lambda, F) \in \Gamma \times \Omega_{\delta, f}$ and

$$
\begin{aligned}
\left\|Q_{\beta}(\lambda) S_{\beta}(\lambda)-Q_{0}(\lambda) S_{0}(\lambda)\right\| \leqq & \left\|\chi_{[-N, N]}\left[Q_{\beta}(\lambda) S_{\beta}(\lambda)-Q_{0}(\lambda) S_{0}(\lambda)\right]\right\| \\
& +\left\|\left(1-\chi_{[-N, N]}\right)\left[Q_{\beta}(\lambda) S_{\beta}(\lambda)-Q_{0}(\lambda) S_{0}(\lambda)\right]\right\| .
\end{aligned}
$$

From (14), there exists a constant $c_{1}(\lambda, F)<\infty$ such that

$$
\left\|\left(1-\chi_{[-N, N]}\right)\left[Q_{\beta}(\lambda) S_{\beta}(\lambda)-Q_{0}(\lambda) S_{0}(\lambda)\right]\right\| \leqq \frac{c_{1}(\lambda, F)}{f^{1 / 2} N^{1 / 2}}\left\|S_{\beta}(\lambda)\right\| \rightarrow 0
$$

as $N \rightarrow \infty$ uniformly in $\beta$ being $\lambda \in \Gamma$, where, by Lemma $3, S_{\beta}(\lambda)$ is uniformly bounded. Moreover, the other term in (24), for $N$ fixed, tends to zero as $\beta \downarrow 0$. In fact, by the resolvent identity, we have:

$$
\begin{aligned}
& \left\|\chi_{[-N, N]}\left(Q_{\beta}(\lambda) S_{\beta}(\lambda)-Q_{0}(\lambda) S_{0}(\lambda)\right)\right\| \\
& \leqq\left\|\chi_{[-N, N]}\left(Q_{\beta}(\lambda)-Q_{0}(\lambda)\right) S_{0}(\lambda)\right\| \\
& +\left\|\chi_{[-N, N]} Q_{\beta}(\lambda) S_{\beta}(\lambda)\left[Q_{\beta}(\lambda)+V_{\beta}^{\theta}-Q_{0}(\lambda)-V_{0}^{\theta}\right] S_{0}(\lambda)\right\| \\
& \leqq c_{2}(\lambda, F) \max _{x \in[-N, N]}\left|Q_{\beta}(\lambda)-Q_{0}(\lambda)\right| \\
& +c_{3}(\lambda, F)\left\|S_{\beta}(\lambda)\right\| \max _{x \in[-N, N]}\left|Q_{\beta}(\lambda)+V_{\beta}^{\theta}-Q_{0}(\lambda)-V_{0}^{\theta}\right| \rightarrow 0
\end{aligned}
$$


as $\beta \downarrow 0$ because of the uniform boundedness of $S_{\beta}(\lambda)$ for $\lambda \in \Gamma$ and from the uniform convergence $V_{\beta}^{\theta} \rightarrow V_{0}^{\theta}$, stated in (11), and $Q_{\beta}(\lambda) \rightarrow Q_{0}(\lambda)$ in any compact set $[-N, N]$, where $c_{2}(\lambda, F)$ and $c_{3}(\lambda, F)$ are two $\beta$-independent constants. Finally, from the uniform boundedness of $S_{\beta}(\lambda)$ in $\Gamma \times \Omega_{\delta, f}$, stated in Lemma 3, and from the uniform convergence, as $\beta \downarrow 0$, of $Q_{\beta}(\lambda)$ and $V_{\beta}^{\theta}$ in $\Gamma \times \Omega_{\delta, f}$ Lemma 4 follows.

Let us stress that, from (13) and from the choice of $\Gamma, \lambda \in \Sigma\left(\hat{H}_{\beta, F}^{\theta}\right) \cap \Gamma$ if and only if $Q_{\beta}(\lambda) S_{\beta}(\lambda)$ has eigenvalue -1 . Now, since $Q_{0}(\lambda) S_{0}(\lambda)$ has simple eigenvalue -1 for $\mu_{j}^{0, F}=\lambda_{j}^{0, F}+i F \theta, j=1, \ldots, n$, then for any $\varepsilon>0$ there exists $\bar{\beta}>0$ such that the eigenprojection

$$
P_{\beta}=-\frac{1}{2 \pi i} \oint_{|z+1|=\varepsilon}\left[Q_{\beta}\left(\mu_{j}^{0, F}\right) S_{\beta}\left(\mu_{j}^{0, F}\right)-z\right]^{-1} d z
$$

has dimension one for any $\beta$ in $[0, \bar{\beta}]$. Indeed $\left[Q_{\beta}\left(\mu_{j}^{0, F}\right) S_{\beta}\left(\mu_{j}^{0, F}\right)-z\right]^{-1}$ converges in norm to $\left[Q_{0}\left(\mu_{j}^{0, F}\right) S_{0}\left(\mu_{j}^{0, F}\right)-z\right]^{-1}$ from Lemma 4 and since $\left[Q_{0}\left(\mu_{j}^{0, F}\right) S_{0}\left(\mu_{j}^{0, F}\right)-z\right]^{-1}$ exists and it is bounded (see, e.g., Kato (1976) Theorem IV-2.23). Then there exists a unique simple eigenvalue $z=z\left(\mu_{j}^{0, F}, \beta\right)$ of $Q_{\beta}\left(\mu_{j}^{0, F}\right) S_{\beta}\left(\mu_{j}^{0, F}\right)$ in the disk $D_{\varepsilon}(-1)$ of radius $\varepsilon$ and center -1 .

Moreover, since $\left\{Q_{\beta}(\lambda) S_{\beta}(\lambda)\right\}_{\lambda}$ is an analytic family of operators in $\lambda \in \Gamma$ and converges in norm as $\beta \downarrow 0$ uniformly in $\Gamma \times \Omega_{\delta, f}$, the function $z_{j}:=z_{j}(\lambda, \beta)$ defined, for $\lambda$ close to $\mu_{j}^{0, F}$ and $\beta$ close to 0 , as the unique simple eigenvalue of the operator $Q_{\beta}(\lambda) S_{\beta}(\lambda)$ in the disk $D_{\varepsilon}(-1)$, is well defined and it is analytic in $\lambda$ and continuous in $\beta$. Besides $z_{j}\left(\mu_{j}^{0, F}, 0\right)=-1$, hence for any $d_{2}>0$ the analytic implicit function theorem, as in Markushevich (1970) IV- $\$ 5.5$, applied to the function $f_{j}(\lambda, \beta):=$ $z_{j}(\lambda, \beta)+1$, assures the existence of $1 \leqq N(j)<\infty$ zeroes $\lambda_{j, i}^{\beta, F}, i=1,2, \ldots, N(j)$, of the function $f_{j}(\lambda, \beta)$ close to $\mu_{j}^{0, F}$ up to $\bar{d}_{2}$ if $\beta \in[0, \bar{\beta}], \bar{\beta}>0$ suitable (here $N=N(j)$ is the minimum positive integer such that $\frac{\partial^{N} z_{j}\left(\mu_{j}^{0, F}, 0\right)}{\partial \lambda^{N}} \neq 0$, since $f_{j}(\lambda, \beta)$ is analytic in $\lambda$ and not identically zero, then $N<\infty)$. Let $\lambda_{j}^{\beta, F}:=\lambda_{j, 1}^{\beta, F}-i F \theta$.

Hence, from the above result and from the uniformity for $F \in \Omega_{\delta, f}$ stated in Lemma 4, Theorem 2 follows.

Now, linking Theorem 1 and Theorem 2, it follows:

Corollary 5. Let $\theta$, with $0<\theta<\theta_{0}$, be fixed and $\delta>0$ as in Theorem $1 i$. Then, for any $d>0$ and for any $f>0$ there exists $\bar{\beta}>0$ such that, $\forall F \in \Omega_{\delta, f}$ and $\forall \beta \in[0, \bar{\beta}]$, $P$-a.s. there exists an eigenvalue $\lambda_{j}^{\beta, F}+F \beta^{-1} X_{m}(\omega)$ at least, for some $m$, of $H_{\beta, F}^{\theta}$ where $\left|\lambda_{j}^{\beta, F}-\lambda_{j}\right|<d, j=1,2, \ldots, n$.

Now, uniqueness and analytical properties will follow from stability arguments as $\beta \downarrow 0$ uniformly for $F$ in $\Theta_{\varepsilon, f}:=\{F \in \mathbf{C}|f \leqq| F \mid \leqq 2 f, \varepsilon \leqq \arg F \leqq \pi / 2\}$.

Theorem 6. For any $\varepsilon>0$ the eigenvalue $\lambda_{j}^{\beta, F}+F \beta^{-1} X_{m}(\omega)$ given in Corollary 5 $(j=1,2, \ldots, n, m \in \mathbf{Z}$ and fixed $)$ is unique and analytic for $F \in \Omega_{\pi / 2, f}$.

Proof of Theorem 6. The assertion follows from a stability argument as $\beta \downarrow 0$ uniform for $F \in \Theta_{\varepsilon, f}$. Here $0<\varepsilon<\delta$, so that the interior of the set $\Theta_{\varepsilon, f} \cap \Omega_{\delta, f}$ is not empty.

Now, we compare $H_{\beta, F}^{\theta}$ with the Stark operator $H_{0, F}^{\theta}=p^{2}+V_{0}^{\theta}+F x-i F \theta$ in the limit $\beta \downarrow 0$. First notice that, as in Theorem $1, H_{0, F}^{\theta}$ admits $n \theta$-independent eigenvalues $\lambda_{j}^{0, F}$ close to $\lambda_{j}$ for $\varepsilon \leqq \arg F \leqq \pi / 2,0<|F| \leqq 2 F_{0}$, for any $\varepsilon>0$, by a stability 
argument as $F \rightarrow 0$ (see, e.g., Vock-Hunziker's criterion in Hunziker (1988)). Moreover, such eigenvalues are analytic in the same region of $F$ since $H_{0, F}^{\theta}$ is an analytic family of operators in $F, \Im F>0$, and they are free of crossings by stability.

Now, fixing $F$ in $\Theta_{\varepsilon, f}$, stability arguments as above work as $\beta \downarrow 0$, too. Indeed, we $P$-a.s. have that

$$
\left\|\left(H_{\beta, F}^{\theta}-H_{0, F}^{\theta}\right) g\right\|-0, \quad \text { as } \beta \downarrow 0, \forall g \in C_{0}^{\infty}(\mathbf{R}) .
$$

Besides, the asymptotic numerical ranges

$$
\left\{\left\langle g, H_{\beta, F}^{\theta} g\right\rangle \mid g \in C_{0}^{\infty}(x>M)\right\} \text { and }\left\{\left\langle g, H_{\beta, F}^{\theta} g\right\rangle \mid g \in C_{0}^{\infty}(x<-M)\right\} \text {, }
$$

for $M$ large, lie far away from the region of eigenvalues since the $F x$ term is dominant with respect to $V_{\beta}^{\theta}$ by the estimate (7). Therefore, for $F \in \Theta_{\varepsilon, f}$ we have only one eigenvalue $\lambda_{j}^{\beta, F}$ close to $\lambda_{j}(j=1,2, \ldots, n)$ and it is the by analytic continuation of the one given in the set $\Omega_{\delta, f}$ since the interior of the set $\Theta_{\varepsilon, f} \cap \Omega_{\delta, f}$ is not empty.

Remark 7. Therefore, a kind of disordered ladder of eigenvalues of $H_{\beta, F}^{\theta} P$-a.s. exists if $\beta^{-1}$ is large enough: for each $j, j=1,2, \ldots, n$, the position of such eigenvalues on the ladder corresponds to the direct image of the sample parameter in the form $\left\{\lambda_{j}^{\beta, F}+\beta^{-1} F X_{m}(\omega)\right\} m \in S_{\bar{\beta}}(\omega) \subset Z$. In particular, from Theorem 6 and from Corollary 2.2 of Herbst and Howland (1981) the existence $P$-a.s. follows, for $\beta<\bar{\beta}$ and $F \in \Omega_{\delta, f}$ and real, of disordered ladders of resonances

$$
\lambda_{j}^{\beta, F}+\beta^{-1} F X_{m}(\omega), \quad \text { where } P\left(m \in S_{\bar{\beta}}(\omega)\right)=1-O(\bar{\beta})
$$

for the Stark-Wannier operator

$$
H_{\beta, F}:=p^{2}+V_{\beta}+F x, \quad V_{\beta}=\sum_{m \in \mathbf{Z}} u\left(x-\beta^{-1} X_{m}(\omega)\right) .
$$

\section{Stark-Wannier States for Crystals}

In this section we specialize the results of the previous section to the case of the onedimensional crystal. In such a case we have that $X_{m}=m a$, where $a$ is the period of the crystal, so that overlapping in (5) is excluded. Hence $u_{\beta}(x-i \theta)$ for $|\theta|<\theta_{0}$ is bounded and $\lim u_{\beta}\left(x-i \theta+\beta^{-1} m a\right)=u(x-i \theta)$ for any $m$ and any $x$. Moreover, the $\beta \downarrow 0$

statements in Corollary 5 and Theorem 6 hold too. Hence the existence, uniqueness and analyticity of the Stark-Wannier ladder $\lambda_{j}^{\beta, F}+m F \beta^{-1} a, m \in \mathbf{Z}$, are given for $F \in \Omega_{\pi / 2, f}$.

However, in the crystal case we can improve the above results because the existence and analyticity of the Stark-Wannier states for complex $F$ in a small disk tangent to the real axis is known. Indeed, the existence and analyticity of a StarkWannier ladder associated with the single-band approximation ladder of Wannier, via a regular perturbation theory, for complex electric field in a disk $D_{\rho}(i \rho)$ of radius $\rho$, tangent to the real axis in the origin, was proved in Bentosela et al. (1988). Note that the radius $\rho$ does not depend on the period of the crystal but, essentially, on the isolation distance of the band.

Hence, with such restrictions, we can take complex values of the electric field arbitrarily small. Moreover the Stark-Wannier states, given by exact ladders, are 
associated by perturbation theory with the single-band approximation states of Wannier.

From a well known stability result, the $j^{\text {th }}$ band tends to $\lambda_{j}$ and the isolation distance of the band tends to the isolation distance of the eigenvalue $\lambda_{j}$ as $\beta \downarrow 0$. Let $r$ be the minimum isolation distance of the first eigenvalues. Choosing $d$ in Corollary 5 such that $d<(r / 4) \sin \delta$ and $f$ so small that $I:=D_{\rho}(i \rho) \cap \Omega_{\delta, f} \cap \Theta_{\delta / 2, f} \neq$ $\varnothing$, then, for any $F \in I$ and $\beta$ small enough, $\left|\lambda_{j}^{\beta, F}-\lambda_{j}\right|<d$. So the ladder $\lambda_{j}^{\beta, F}+m \beta^{-1} F a$ is contained in the strip with boundaries $\left(\lambda_{j}-r / 2\right)+F \mathbf{R}$ and $\left(\lambda_{j}+r / 2\right)+F \mathbf{R}$. Hence, from the uniqueness condition stated in Bentosela et al. (1988), this ladder must be unique and must coincide with the Stark-Wannier ladder associated with the singleband approximation. In particular, since $H_{\beta, F}^{\theta}$ is an analytical family for $F \in \mathbf{C}^{+}:=$ $\{z \in \mathbf{C} \mid \mathfrak{I} z>0\}$ and the eigenvalues $\lambda_{j}^{\beta, F}$ are isolated for $F \in \Omega_{\delta, f}$, then a unique analytic continuation from the disk $D_{\rho}(i \rho)$ to the real axis for the Stark-Wannier ladders is given.

We can summarize these results in the following:

Theorem 8. Let $H_{\beta, F}$ be the Stark-Wannier operator

$$
H_{\beta, F}:=p^{2}+V_{\beta}+F x, \quad V_{\beta}=\sum_{m \in \mathbf{Z}} u\left(x-m a \beta^{-1}\right) \text {. }
$$

Then, for any $f>0, f<F_{0}, H_{\beta, F}$ has ladders of resonances for $\beta>0$ small enough and any real $F$ in $[f, 2 f]$. Such resonances are the limit, as $\mathfrak{I} F \downarrow 0$, of the complex $F$ Stark-Wannier states uniquely associated with the single-band Wannier states.

We conclude this paper considering a model coincident, for $F=0$, with the classical Lamé problem. Let

$$
V_{k}^{\theta}(x):=v \cdot(v+1)\left[k^{2} \operatorname{sn}^{2}(x-i \theta, k)-1\right], \quad k \leqq 1,|\theta|<\theta_{0}=\pi / 2, v \geqq 1,
$$

where $\operatorname{sn}(z, k)$ is the Jacobian elliptic function having real period $4 K$ and imaginary period $2 i K^{\prime}$. In such a model the role of $\beta$ is played by $K^{-1}$. Here $K$ and $K^{\prime}$ are functions of $k$ given by

$$
K(k)=\int_{0}^{\pi / 2} \frac{d \phi}{\sqrt{1-k^{2} \sin ^{2} \phi}} \text { and } K^{\prime}(k)=K\left(\sqrt{1-k^{2}}\right) .
$$

In particular, we have that

$$
\lim _{k \uparrow 1} K(k)=+\infty \quad \text { and } \quad \lim _{k \uparrow 0} K^{\prime}(k)=\pi / 2
$$

Moreover the following formula holds:

$$
\operatorname{sn}^{2}(z, k)=\left(\frac{\pi}{2 K^{\prime}}\right)^{2}\left\{\sum_{m=-\infty}^{+\infty}\left[\cosh ^{-2}\left(\frac{m K \pi}{K^{\prime}}\right)-\cosh ^{-2}\left(z \frac{\pi}{2 K^{\prime}}-K X_{m}\right)\right]\right\},
$$

$X_{m}=\frac{m \pi}{K^{\prime}}$. Equation (36) follows from the formula given in Whittaker and Watson (1965), Chap. 20, Sect. 2, for the Weierstrassian elliptic function $\mathscr{P}(z)$ (with real period $2 K$ and imaginary period $\left.i 2 K^{\prime}\right)$ and identifying $\mathscr{P}\left(z+i K^{\prime}\right)$ with $\operatorname{sn}^{2}(z, k)$ up to an additive constant. From (36) it follows immediately that as $k \uparrow 1$ (i.e. as $K \rightarrow \infty$ ) each $m \neq 0$ term tends to zero, hence, for any $x$, we have $V_{k}^{\theta}(x) \rightarrow v$. 
$(v+1)\left[\tanh ^{2}(x-i \theta)-1\right]$ as $k \uparrow 1$. The discrete spectrum of

$$
H=p^{2}+v \cdot(v+1)\left[\tanh ^{2}(x)-1\right]
$$

is not empty for $v>0$ and consists (see, e.g., Sect. 12.3 Morse and Feshbach (1953)) of $n$ non-degenerate eigenvalues

$$
\lambda_{j}=-(v-j+1)^{2}, \quad j=1, \ldots, n=-[-v],
$$

where $[\cdot]$ denotes the integral part.

Acknowledgements. It is pleasure to thank Professor François Bentosela for many discussions on such problems. This work is partially supported by Ministero dell'Università e della Ricerca Scientifica e Tecnologica.

\section{References}

1. Agler, J., Froese, R.: Existence of Stark ladder resonances. Commun. Math. Phys. 100, 161-171 (1985)

2. Avron, J.: On the spectrum of $p^{2}+V(x)+\varepsilon x$, with $V$ periodic and $\varepsilon$ complex. J. Phys. A: Math. Gen. 12, 2393-2398 (1979)

3. Avron, J.: The lifetime of Wannier ladder states. Ann. Phys. 143, 33-53 (1982)

4. Bentosela, F., Caliceti, E., Grecchi, V., Maioli, M., Sacchetti, A.: Analyticity and asymptotics for the Stark-Wannier states. J. Phys. A: Math. Gen. 21, 3321-3331 (1988)

5. Bentosela, F., Grecchi, V.: Stark-Wannier ladders. Commun. Math. Phys. 142, 169-192(1991)

6. Buslaev, V.S., Dmitrieva, L.A.: A Bloch electron in an external field. Leningrad Math. J. 1, 287-320 (1990)

7. Combes, J.M., Hislop, P.D.: Stark ladder resonances for small electric fields. Commun. Math. Phys. 140, 291-320 (1991)

8. Herbst, I.: Dilation Analyticity in constant electric field: I. The two body problem. Commun. Math. Phys. 64, 279-298 (1979)

9. Herbst, I., Howland, J.: The Stark ladder and other one-dimensional external electric field problems. Commun. Math. Phys. 80, 23-42 (1981)

10. Hunziker, W.: Notes on asymptotic perturbation theory for Schrödinger eigenvalue problems. Helv. Phys. Acta 61, 257-304 (1988)

11. Kato, T.: Perturbation theory for linear operator. Berlin, Heidelberg, New York: Springer 1976

12. Kirsch, W., Kotani, S., Simon, B.: Absence of absolutely continuous spectrum for some one dimensional random but deterministic Schrödinger operators. Ann. Inst. Henri Poincarè 42, 383-406 (1985)

13. Markushevich, A.: Teoria de las funciones analiticas. MIR, 1970

14. Morse, P.M., Feshbach, H.: Methods of theoretical Physics, vol. II. New York: McGraw-Hill 1953

15. Nenciu, A., Nenciu, G.: Existence of Stark-Wannier resonances for non-periodic onedimensional systems. Phys. Rev. B 40, 3622-3624 (1989)

16. Sigal, I.M.: Sharp exponential bounds on resonances states and width of resonances. Adv. Appl. Math. 9, 127-166 (1988)

17. Whittaker, E.T., Watson, G.N.: A course of modern analysis. Cambridge Univ. Press 1965 